\title{
Quantum Self-Frictional Relativistic Nucleoseed Spinor-Type Tensor Field Theory of Nature
}

\author{
I. I. Guseinov \\ Department of Physics, Faculty of Arts and Sciences, Onsekiz Mart University, Çanakkale, Turkey \\ Correspondence should be addressed to I. I. Guseinov; isguseinov@yahoo.com
}

Received 3 December 2016; Revised 16 January 2017; Accepted 19 January 2017; Published 3 April 2017

Academic Editor: Shi-Hai Dong

Copyright (C) 2017 I. I. Guseinov. This is an open access article distributed under the Creative Commons Attribution License, which permits unrestricted use, distribution, and reproduction in any medium, provided the original work is properly cited. The publication of this article was funded by $\mathrm{SCOAP}^{3}$.

\begin{abstract}
For study of quantum self-frictional (SF) relativistic nucleoseed spinor-type tensor (NSST) field theory of nature (SF-NSST atomicmolecular-nuclear and cosmic-universe systems) we use the complete orthogonal basis sets of $2(2 s+1)$-component columnmatrices type SF $\Psi_{n l j m_{j}}^{\left(\delta^{*}\right) s}$-relativistic NSST orbitals $\left(\Psi^{\left(\delta^{*}\right) s}\right.$-RNSSTO) and SF $X_{n l j m_{j}}^{s}$-relativistic Slater NSST orbitals $\left(X^{s}\right.$-RSNSSTO) through the $\psi_{n l m_{l}}^{\left(\delta^{*}\right)}$-nonrelativistic scalar orbitals $\left(\psi^{\left(\delta^{*}\right)}\right.$-NSO) and $\chi_{n l m_{l}}$-nonrelativistic Slater type orbitals $(\chi$-NSTO), respectively. Here $\delta^{*}=p_{l}^{*}$ or $\delta^{*}=\alpha^{*}$ and $p_{l}^{*}=2 l+2-\alpha^{*}, \alpha^{*}$ are the integer $\left(\alpha^{*}=\alpha,-\infty<\alpha \leq 2\right)$ or noninteger $\left(\alpha^{*} \neq \alpha,-\infty<\alpha^{*}<3\right)$ SF quantum numbers, where $s=0,1 / 2,1,3 / 2,2, \ldots$ We notice that the nonrelativistic $\psi^{\left(\delta^{*}\right)}$-NSO and $\chi$-NSTO orbitals themselves are obtained from the relativistic $\Psi^{\left(\delta^{*}\right) s}$-RNSSTO and $X^{s}$-RSNSSTO functions for $s=0$, respectively. The column-matrices-type SF ${ }^{1} Y_{j m_{j}}^{l s}$-RNSST harmonics $\left({ }^{1} Y^{l s}-\right.$ RNSSTH $)$ and ${ }^{2} Y_{j m_{j}}^{l_{s}}$-modified NSSTH $\left({ }^{2} Y^{l s}\right.$-MNSSTH) functions for arbitrary spin $s$ introduced by the author in the previous papers are also used. The one- and two-center one-range addition theorems for $\psi^{\left(\delta^{*}\right)}$-NSO and noninteger $n \chi$-NSTO orbitals are presented. The quantum SF relativistic nonperturbative theory for $V_{n l j m_{j}}^{\left(\delta^{*}\right)}$-RNSST potentials $\left(V^{\left(\delta^{*}\right)}\right.$-RNSSTP) and their derivatives is also suggested. To study the transportations of mass and momentum in nature the quantum SF relativistic NSST gravitational photon (gph) with $s=1$ is introduced.
\end{abstract}

\section{Introduction}

Construction of combined quantum approach for AMN and $\mathrm{CU}$ systems of nature is the most important because the classical aspect of field theories leads to contradictions (see review papers $[1,2]$ and references therein). These contradictions, as shown in Figures 1 and 2 for nonrelativistic and SF relativistic NSST potentials and forces, arise for some values of distance $r\left(10^{-16} \leq r \leq 10^{-15}, 10^{-14} \leq r \leq 10^{-13}, 10^{-12} \leq\right.$ $r \leq 10^{-11}, 10^{-9} \leq r \leq 10^{-8}$, and $10^{-6} \leq r \leq 10^{-5}$ ) between fields of nature. The quantities $G,\left(N_{1}\right.$ and $\left.N_{2}\right), E, N W$, and NUA in Figures 1 and 2 describe the gravitational, nuclear, electromagnetic, nuclear weak, and Newtonian (Newtonian universal attraction law) fields, respectively (see $[3,4]$ on observation of gravitational $(G)$ and nuclear $\left(N_{1}\right.$ and $\left.N_{2}\right)$ fields). The difficulties arising for these values of $r$ are not explained by classical field theories. Taking into account all values of distance from nucleus (for $0<r<\infty$ ), such a problem can be solved only using quantum SF relativistic NSST field theory of nature presented in this work. We note that the $G, N, E$, NW, and NUA fields are obtained from the single quantum SF relativistic NSST field when the SF properties are disappearing from sight.

According to the theory introduced by Lorentz in classical electrodynamics [5-7], the electrons move around the atomic point-charge nuclei under total nuclear attraction forces $\vec{F}_{L}=$ $\vec{F}+\left(2 e^{2} / 3 c^{3}\right) \dddot{\vec{r}}$, where $\ddot{\vec{r}}$ is the time derivative of the acceleration of the electron. We note that the inclusion of the third derivative of displacement leads to the radiation and selfforce problems in classical electrodynamics. These problems do not arise in the case of quantum SF relativistic NSST field 


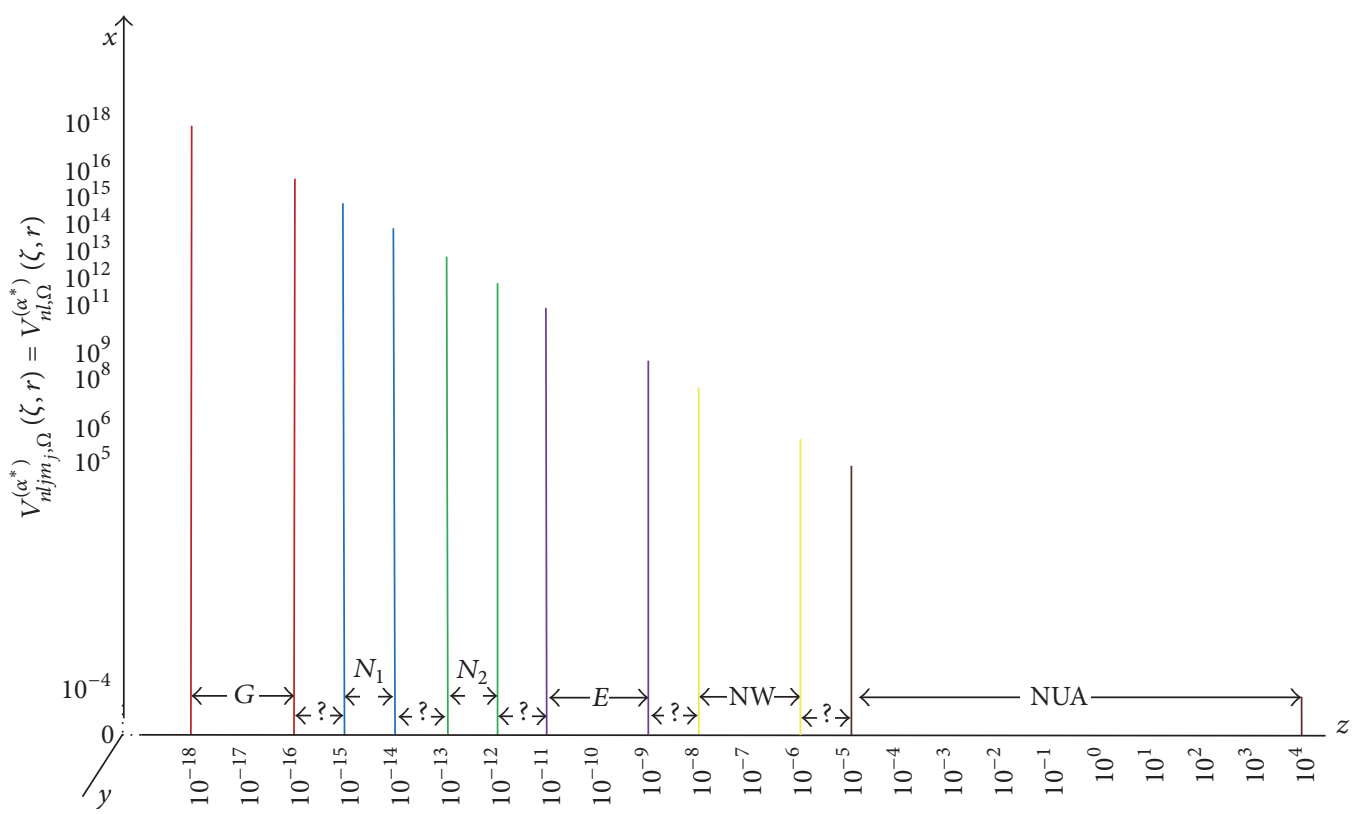

FIGURE 1: Quantum scalar and SF relativistic NSST potentials for $\lambda=0$ as a function of distance $r$ (for $z=r, n=2, l=1, j=1 / 2, m_{j}=-1 / 2$, $\left.\zeta=1.5, \alpha^{*}=0.5\right)$ for $\Omega=G\left(10^{-18} \leq r \leq 10^{-16}\right), \Omega=N_{1}\left(10^{-15} \leq r \leq 10^{-14}\right), \Omega=N_{2}\left(10^{-13} \leq r \leq 10^{-12}\right), \Omega=E\left(10^{-11} \leq r \leq 10^{-9}\right)$, $\Omega=\mathrm{NW}\left(10^{-8} \leq r \leq 10^{-6}\right)$, and $\Omega=\mathrm{NUA}\left(10^{-5} \leq r \leq 10^{4}\right)$.

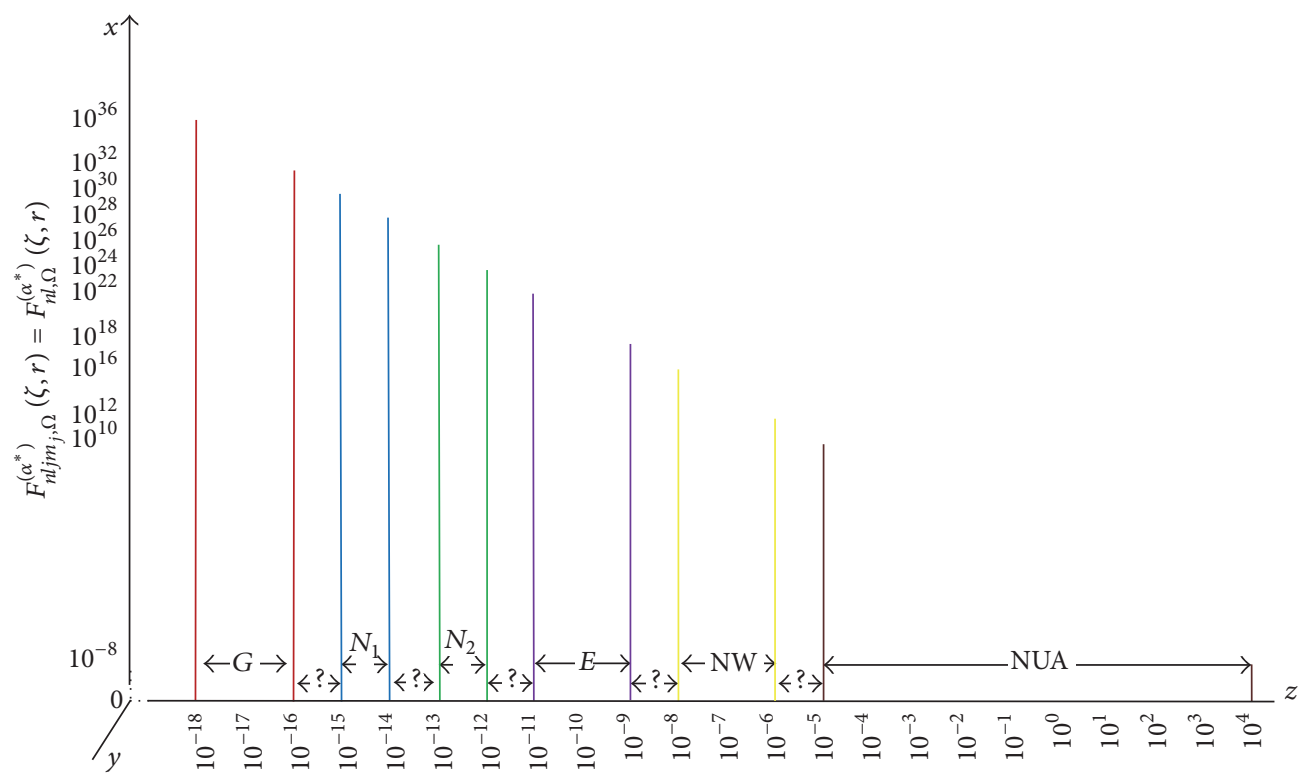

FIgURE 2: Quantum scalar and SF relativistic NSST attraction forces for $\lambda=0$ as a function of distance $r$ (for $z=r, n=2, l=1, j=1 / 2$, $\left.m_{j}=-1 / 2, \zeta=1.5, \alpha^{*}=0.5\right)$ for $\Omega=G\left(10^{-18} \leq r \leq 10^{-16}\right), \Omega=N_{1}\left(10^{-15} \leq r \leq 10^{-14}\right), \Omega=N_{2}\left(10^{-13} \leq r \leq 10^{-12}\right), \Omega=E\left(10^{-11} \leq r \leq\right.$ $\left.10^{-9}\right), \Omega=\mathrm{NW}\left(10^{-8} \leq r \leq 10^{-6}\right)$, and $\Omega=\mathrm{NUA}\left(10^{-5} \leq r \leq 10^{4}\right)$.

theory. The analytical formulas for the quantum attraction forces suggested in the previous papers [8-10] are the extensions of Lorentz theory to the quantum cases in standard and nonstandard conventions (see $[11,12]$ and references therein to our works on standard and nonstandard conventions). In the quantum cases, the SF particles move around the nucleus under relativistic NSST attraction forces. These forces depend on quantum numbers $n, l, \alpha^{*}$ and scaling parameters $\zeta$ and distance $r$ from nucleus of SF-NSST AMN and CU systems. We note that the new fields, which may be discovered in the 
future for $0<r<10^{-18}$, depended on the productive capacity of science and technology. The presented quantum nonrelativistic and SF relativistic NSST field theory of nature is the generalization introduced by the author of AMN approach to the CU systems.

The purpose of this work is to construct the combined quantum nonrelativistic and relativistic NSST field theory of nature in position space for arbitrary values of parameters. This theory may open new avenues of approach to the solution of problems related to the properties of AMN-CU systems.

\section{Gravitational Photon}

To study the quantum SF relativistic NSST field theory of nature, we use the Einstein classical relativistic relation between mass and energy in the following form:

$$
E(m)=m c^{2}
$$

where $m$ (for $0<m<\infty$ ) is the mass of fermion; $m c^{2}$ and $E(m)$ are the energies of fermion and gph boson with $s=1$, respectively. The gph boson moves with the velocity of light $(v=c)$ and carries the mass $m=E(m) / c^{2}$ and momentum $p$. We note that, in the case of classical electrodynamics, the SF properties of fermions and bosons disappear and the radiation problems arise.

\section{Quantum Nonrelativistic Field Theory in Standard Convention}

It is easy to show that the SF relativistic NSST functions are expressed through the corresponding nonrelativistic basis sets. Therefore, the SF relativistic NSST particles can be described by the use of nonrelativistic functions. Now we investigate at first the nonrelativistic cases.

In order to study the scalar quantum field theory of nature in standard convention, we use the following formulas (see [8-10]):

(1) The complete orthogonal sets are as follows:

$$
\begin{aligned}
& \mathscr{L}_{n l}^{\left(\delta^{*}\right)}(t)=\frac{\gamma_{n l}^{\left(\delta^{*}\right)}}{\Gamma\left(p_{l}^{*}+1\right)} t^{l \delta_{\delta^{*} \alpha^{*}}}{ }_{1} F_{1}\left(-[n-(l+1)] ; p_{l}^{*}\right. \\
& +1 ; t) \\
& \mathscr{L}_{n l}^{\left(\delta^{*}\right)}(t)=\gamma_{n l}^{\left(\delta^{*}\right)} \sum_{n^{\prime}=l+1}^{n} \tilde{a}_{n n^{\prime}}^{\left(\delta^{*}\right) l} t^{n^{\prime}-\left(l \delta_{\delta^{*}} p_{l}^{*+1}\right)} \\
& \overline{\mathscr{L}}_{n l}^{\left(\delta^{*}\right)}(t)=\frac{1}{\gamma_{n l}^{\left(p_{l}^{*}\right)}} \mathscr{L}_{n l}^{\left(p_{l}^{*}\right)}(t) \quad \text { for } \delta^{*}=p_{l}^{*} \\
& \overline{\mathscr{L}}_{n l}^{\left(\delta^{*}\right)}(t)=\beta_{n l}^{\left(\alpha^{*}\right)}(t) \mathscr{L}_{n l}^{\left(\alpha^{*}\right)}(t) \quad \text { for } \delta^{*}=\alpha^{*} \\
& \psi_{n l m_{l}}^{\left(\delta^{*}\right)}(\zeta, \vec{r})=(2 \zeta)^{3 / 2} e^{-\zeta r} \mathscr{L}_{n l}^{\left(\delta^{*}\right)}(t) S_{l m_{l}}(\theta, \phi)
\end{aligned}
$$

$$
\begin{aligned}
& \psi_{n l m_{l}}^{\left(\delta^{*}\right)}(\zeta, \vec{r}) \\
& =\gamma_{n l}^{\left(\delta^{*}\right)} \sum_{n^{\prime}=l+1}^{n} \tilde{a}_{n n^{\prime}}^{\left(\delta^{*}\right) l} \sqrt{\left(2\left(n^{\prime}-l \delta_{\delta^{*} p_{l}^{*}}\right)\right) !} \chi_{n^{\prime}-l \delta_{\delta^{*} p_{l}^{*}} l m_{l}}(\zeta, \\
& \vec{r}) \\
& \bar{\psi}_{n l m_{l}}^{\left(\delta^{*}\right)}(\zeta, \vec{r})=(2 \zeta)^{3 / 2} e^{-\zeta r} \overline{\mathscr{L}}_{n l}^{\left(\delta^{*}\right)}(t) S_{l m_{l}}(\theta, \phi)=\beta_{n l}^{\left(\delta^{*}\right)}(t) \\
& \cdot \psi_{n l m_{l}}^{\left(\delta^{*}\right)}(\zeta, \vec{r}) \\
& \bar{\psi}_{n l m_{l}}^{\left(\delta^{*}\right)}(\zeta, \vec{r})=\eta_{n l}^{\left(\delta^{*}\right)}(t) \\
& \cdot \sum_{n^{\prime}=l+1}^{n} \tilde{a}_{n n^{\prime}}^{\left(\delta^{*}\right) l} \sqrt{\left(2\left(n^{\prime}-l \delta_{\delta^{*}} p_{l}^{*}\right)\right) !} \chi_{n^{\prime}-l \delta_{\delta^{*} p_{l}^{*}} \operatorname{lm}_{l}}(\zeta, \vec{r}),
\end{aligned}
$$

where

$$
\begin{aligned}
\chi_{n l m_{l}}(\zeta, \vec{r}) & =\frac{(2 \zeta)^{n+1 / 2}}{\sqrt{(2 n) !}} r^{n-1} e^{-\zeta r} S_{l m_{l}}(\theta, \phi) \\
\beta_{n l}^{\left(\delta^{*}\right)}(t) & =\frac{(n-(l+1)) !}{\Gamma\left(q_{n}^{*}+1\right)} t^{p_{l}^{*}-2} \quad \text { for } \delta^{*}=p_{l}^{*} \\
\beta_{n l}^{\left(\delta^{*}\right)}(t) & =\left(\frac{2 n}{t}\right)^{\alpha^{*}} \text { for } \delta^{*}=\alpha^{*} \\
\gamma_{n l}^{\left(\delta^{*}\right)} & =\frac{\Gamma\left(q_{n}^{*}+1\right)}{(n-(l+1)) !} \text { for } \delta^{*}=p_{l}^{*} \\
\gamma_{n l}^{\left(\delta^{*}\right)} & =\sqrt{\frac{\Gamma\left(q_{n}^{*}+1\right)}{(2 n)^{\alpha^{*}}(n-(l+1)) !} \quad \text { for } \delta^{*}=\alpha^{*}} \\
\eta_{n l}^{\left(\delta^{*}\right)}(t) & =\gamma_{n l}^{\left(\delta^{*}\right)} \beta_{n l}^{\left(\delta^{*}\right)}(t)=t^{p_{l}^{*}-2} \quad \text { for } \delta^{*}=p_{l}^{*} \\
\eta_{n l}^{\left(\delta^{*}\right)}(t) & =\gamma_{n l}^{\left(\delta^{*}\right)} \beta_{n l}^{\left(\delta^{*}\right)}(t)=\frac{1}{t^{\alpha^{*}}} \sqrt{\frac{(2 n)^{\alpha^{*}} \Gamma\left(q_{n}^{*}+1\right)}{(n-(l+1)) !}}
\end{aligned}
$$

and $t=2 \zeta r, p_{l}^{*}=2 l+2-\alpha^{*}, q_{n}^{*}=n+l+1-\alpha^{*}$, and $\mathscr{L}_{n l}^{\left(\delta^{*}\right)}$ are the NSST polynomials ( $\mathscr{L}^{\left(\delta^{*}\right)}$-NSSTP), $\chi_{n l m_{l}}(\zeta, \vec{r})$ are the nonrelativistic Slater type orbitals, and the $S_{l m_{l}}$ is the complex (for $S_{l m_{l}} \equiv Y_{l m_{l}}$ ) or real spherical harmonic (see [13]). We note that our definition of phases for the complex spherical harmonics $\left(Y_{l m_{l}}^{*}=Y_{l-m_{l}}\right)$ differs from the Condon-Shortley phases [14] by the sign factor $(-1)^{m_{l}}$.

The confluent hypergeometric function ${ }_{1} F_{1}$ [15] occurring in $((2 \mathrm{a})$ and $(2 \mathrm{~b}))$ can be determined by

$$
\begin{aligned}
{ }_{1} F_{1} & \left(-[n-(l+1)] ; p_{l}^{*}+1 ; t\right) \\
& =\Gamma\left(p_{l}^{*}+1\right) \sum_{n^{\prime}=l+1}^{n} \widetilde{a}_{n n^{\prime}}^{\left(\delta^{*}\right) l} t^{n^{\prime}-(l+1)},
\end{aligned}
$$


where

$$
\begin{aligned}
\tilde{a}_{n n^{\prime}}^{\left(p_{l}^{*}\right) l} & =\widetilde{a}_{n n^{\prime}}^{\left(\alpha^{*}\right) l} \\
& =\frac{(-[n-(l+1)])_{n^{\prime}-(l+1)}}{\Gamma\left(p_{l}^{*}+1\right)\left(p_{l}^{*}+1\right)_{n^{\prime}-(l+1)}\left(n^{\prime}-(l+1)\right) !} .
\end{aligned}
$$

As we see, all of the functions $\psi_{n l m_{l}}^{\left(\delta^{*}\right)}(\zeta, \vec{r}), \bar{\psi}_{n l m_{l}}^{\left(\delta^{*}\right)}(\zeta, \vec{r}), \mathscr{L}_{n l}^{\left(\delta^{*}\right)}(t)$, and $\overline{\mathscr{L}}_{n l}^{\left(\delta^{*}\right)}(t)$ are expressed through the Pochhammer symbols.

The orthogonality relations are defined as

$$
\begin{aligned}
& \int \psi_{n l m_{l}}^{\left(\delta^{*}\right)^{*}}(\zeta, \vec{r}) \bar{\psi}_{n^{\prime} l^{\prime} m_{l}^{\prime}}^{\left.\delta^{*}\right)}(\zeta, \vec{r}) d^{3} \vec{r}=\delta_{n n^{\prime}} \delta_{l l^{\prime}} \delta_{m_{l} m_{l}^{\prime}} \\
& \int_{0}^{\infty} e^{-t}\left(t^{p_{l}^{*}-2}\right)^{\delta_{\delta^{*} p_{l}^{*}}}\left(t \beta_{n l}^{\left(\delta^{*}\right)}(t)\right) \mathscr{L}_{n l}^{\left(\delta^{*}\right)}(t)\left(\frac{t}{\beta_{n^{\prime} l}^{\left(\delta^{*}\right)}(t)}\right) \\
& \cdot \overline{\mathscr{L}}_{n^{\prime} l}^{\left(\delta^{*}\right)}(t) d t=\delta_{n n^{\prime}} \\
& \int \chi_{n l m_{l}}^{*}(\zeta, \vec{r}) \chi_{n^{\prime} l^{\prime} m_{l}^{\prime}}(\zeta, \vec{r}) d^{3} \vec{r}=\frac{\left(n+n^{\prime}\right) !}{\sqrt{(2 n) !\left(2 n^{\prime}\right) !}} \\
& \cdot \delta_{l l^{\prime}} \delta_{m_{l} m_{l}^{\prime}}
\end{aligned}
$$

(2) The eigenvalues corresponding to the $\psi_{n l m_{l}}^{\left(\delta^{*}\right)}(\zeta, \vec{r})$ scalar functions are the same and determined by

$$
E^{\left(\delta^{*}\right)}=E=-\frac{\zeta^{2}}{2}
$$

where $\zeta(0<\zeta<\infty)$ is the screening constant. We note that the parameters $\zeta$ can be chosen properly according to the nature of corresponding field under consideration.

(3) The scalar potentials are as follows:

$$
\begin{aligned}
V_{n l}^{\left(\delta^{*}\right)}(\zeta, r) & =-\frac{\zeta n}{r} U_{n l}^{\left(\delta^{*}\right)}(t) \\
U_{n l}^{\left(\delta^{*}\right)}(t) & =1+\frac{\alpha^{*}-1}{n} q_{n}^{*} \frac{\mathscr{L}_{n-1 l}^{\left(p_{l}^{*}+1\right)}(t)}{\mathscr{L}_{n l}^{\left(p_{l}^{*}\right)}(t)}
\end{aligned}
$$

$$
\text { for } \delta^{*}=p_{l}^{*}
$$

$$
U_{n l}^{\left(\delta^{*}\right)}(t)=1+\frac{\alpha^{*}-1}{n} \sqrt{2 n(n-(l+1))} \frac{\mathscr{L}_{n l+1}^{\left(\alpha^{*}+1\right)}(t)}{t \mathscr{L}_{n l}^{\left(\alpha^{*}\right)}(t)}
$$

$$
\text { for } \delta^{*}=\alpha^{*} \text {. }
$$

(4) The scalar forces are as follows:

$$
\begin{aligned}
& F_{n l}^{\left(\delta^{*}\right)}(\zeta, r)=-\frac{\zeta n}{r^{2}} G_{n l}^{\left(\delta^{*}\right)}(t) \\
& G_{n l}^{\left(\delta^{*}\right)}(t)=1+\frac{\alpha^{*}-1}{n} q_{n}^{*} \frac{\mathscr{L}_{n-1 l}^{\left(p_{l}^{*}+1\right)}(t)}{\mathscr{L}_{n l}^{\left(p_{l}^{*}\right)}(t)}\{1 \\
& \left.+t\left[q_{n}^{*} \frac{\mathscr{L}_{n-1 l}^{\left(p_{l}^{*}+1\right)}(t)}{\mathscr{L}_{n l}^{\left(p_{l}^{*}\right)}(t)}-\left(q_{n}^{*}-1\right) \frac{\mathscr{L}_{n-2 l}^{\left(p_{p}^{*}+2\right)}(t)}{\mathscr{L}_{n-1 l}^{\left(p_{l}^{*}+1\right)}(t)}\right]\right\}
\end{aligned}
$$

$$
\text { for } \delta^{*}=p_{l}^{*}
$$

$$
\begin{gathered}
G_{n l}^{\left(\delta^{*}\right)}(t)=1-\frac{\alpha^{*}-1}{n}(n-(l+1)) \times\{1 \\
-\frac{\mathscr{L}_{n l+1}^{\left(\alpha^{*}+1\right)}(t)}{t \mathscr{L}_{n l}^{\left(\alpha^{*}\right)}(t)}\left[\sqrt{\frac{2 n}{n-(l+1)}}\left(p_{l}^{*}+2-t\right)\right. \\
\left.\left.-(2 n) \frac{\mathscr{L}_{n l+1}^{\left(\alpha^{*}+1\right)}(t)}{\mathscr{L}_{n l}^{\left(\alpha^{*}\right)}(t)}\right]\right\} \text { for } \delta^{*}=\alpha^{*} .
\end{gathered}
$$

(5) The one- and two-center one-range addition theorems for nonrelativistic $\psi^{\left(\delta^{*}\right)}$-NSO and noninteger $n \chi$-NSTO functions in standard convention are determined by the following relations.

$\psi^{\left(\delta^{*}\right)}$-NSO Functions. For $\vec{R}_{a b} \neq 0$

$$
\begin{aligned}
& \psi_{n l m_{l}}^{\left(\delta^{*}\right)}\left(\zeta, \vec{r}_{a}\right) \\
& =\sum_{\mu=\nu+1}^{\infty} \sum_{\nu=0}^{\mu-1} \sum_{\sigma=-\nu}^{\nu} \widetilde{\Lambda}_{\mu \nu \sigma, n l m_{l}}^{\left(\delta^{*}\right) v}\left(\zeta, \zeta^{\prime} ; \vec{R}_{a b}\right) \psi_{\mu \nu \sigma}^{\left(\delta^{*}\right)}\left(\zeta^{\prime}, \vec{r}_{b}\right),
\end{aligned}
$$

where

$$
\begin{aligned}
& \widetilde{\Lambda}_{\mu \nu \sigma, n l m_{l}}^{\left(\delta^{*}\right) v}\left(\zeta, \zeta^{\prime} ; \vec{R}_{a b}\right)=\sum_{n^{\prime}=l+1}^{n} \sum_{\mu^{\prime}=\nu+1}^{\mu} \widetilde{\Omega}_{n l, n^{\prime} l^{\prime}}^{\left(\delta^{*}\right) l} \widetilde{\Omega}_{\mu \nu, \mu^{\prime} \nu}^{\left(\delta^{*}\right) \nu} \tau_{\mu \nu, \mu^{\prime} \nu}^{\left(\delta^{*}\right) \nu} S_{\left(n^{\prime}-l \delta_{\delta^{*} p_{l}^{*}}^{*}\right) l m_{l},\left(\mu^{\prime}+v \delta_{\left.\delta^{*} p_{l}^{*}-\alpha^{*}\right) \nu \sigma}\right.}\left(\zeta, \zeta^{\prime} ; \vec{R}_{a b}\right) \\
& \tau_{\mu \nu, \mu^{\prime} \nu}^{\left(\delta^{*}\right) \nu}=\frac{(\mu-(\nu+1)) !}{\Gamma\left(q_{\mu}^{*}+1\right)} \sqrt{\frac{\Gamma\left(2\left(\mu^{\prime}+\nu-\alpha^{*}\right)+1\right)}{\left(2\left(\mu^{\prime}-\nu\right)\right) !}} \text { for } \delta^{*}=p_{l}^{*} \\
& \tau_{\mu \nu, \mu^{\prime} \nu}^{\left(\delta^{*}\right) v}=(2 \mu)^{\alpha^{*}} \sqrt{\frac{\Gamma\left(2\left(\mu^{\prime}-\alpha^{*}\right)+1\right)}{\left(2 \mu^{\prime}\right) !}} \text { for } \delta^{*}=\alpha^{*}
\end{aligned}
$$




$$
\begin{aligned}
\widetilde{\Omega}_{n l, n^{\prime} l}^{\left(\delta^{*}\right) l} & =\gamma_{n l}^{\left(\delta^{*}\right)} \widetilde{a}_{n n^{\prime}}^{\left(\delta^{*}\right) l} \sqrt{\left(2\left(n^{\prime}-l \delta_{\delta^{*} p_{l}^{*}}\right)\right) !} \\
S_{n l m_{l}, n^{*} v \sigma}^{*}\left(\zeta, \zeta^{\prime} ; \vec{R}_{a b}\right) & =\int \chi_{n l m_{l}}^{*}\left(\zeta, \vec{r}_{a}\right) \chi_{n^{*} v \sigma}\left(\zeta^{\prime}, \vec{r}_{b}\right) d^{3} \vec{r} .
\end{aligned}
$$

For $\vec{R}_{a b}=0$

$$
\begin{aligned}
& \psi_{n l m_{l}}^{\left(\delta^{*}\right)}(\zeta, \vec{r})=\sum_{\mu=l+1}^{\infty} \widetilde{\Lambda}_{\mu l m_{l}, n l m_{l}}^{\left(\delta^{*}\right) l}\left(\zeta, \zeta^{\prime}\right) \psi_{\mu l m_{l}}^{\left(\delta^{*}\right)}\left(\zeta^{\prime}, \vec{r}\right) \\
& \widetilde{\Lambda}_{\mu l m_{l}, n^{*} l m_{l}}^{\left(\delta^{*}\right) l}\left(\zeta, \zeta^{\prime}\right)=\delta_{\mu n} \quad \text { for } \zeta=\zeta^{\prime} \\
& \widetilde{\Lambda}_{\mu l m_{l}, n^{*} l m_{l}}^{\left(\delta^{*}\right) l}\left(\zeta, \zeta^{\prime}\right)
\end{aligned}
$$

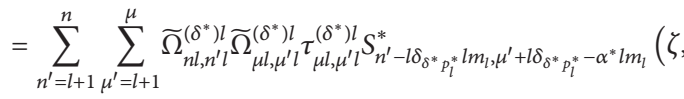

$$
\begin{aligned}
& \left.\zeta^{\prime}\right) \text { for } \zeta \neq \zeta^{\prime} \\
& S_{n l m_{l}, n^{*} v \sigma}^{*}\left(\zeta, \zeta^{\prime}\right)=\delta_{\nu l} \delta_{\sigma m_{l}} \frac{\Gamma\left(n+n^{*}+1\right)}{\sqrt{(2 n) ! \Gamma\left(2 n^{*}+1\right)}}(1+t)^{n+1 / 2}(1 \\
& -t)^{n^{*}+1 / 2} \\
& t=\frac{\zeta-\zeta^{\prime}}{\zeta+\zeta^{\prime}}
\end{aligned}
$$

See [16] for the calculation of overlap integrals.

Noninteger $n \chi$-NSTO Functions. For $\vec{R}_{a b} \neq 0$

$$
\begin{aligned}
& \chi_{n^{*} l m_{l}}\left(\zeta, \vec{r}_{a}\right) \\
& =\sum_{\mu=\nu+1}^{\infty} \sum_{u=v+1}^{\mu} \sum_{\nu=0}^{\mu-1} \sum_{\sigma=-\nu}^{\nu} \widetilde{\Pi}_{\mu u, v \sigma, n^{*} l m_{l}}^{\left(\delta^{*}\right) v}\left(\zeta, \zeta^{\prime} ; \vec{R}_{a b}\right) \chi_{u-v \delta_{\delta^{*} p_{\nu}^{*} v \sigma}}\left(\zeta^{\prime}, \vec{r}_{b}\right),
\end{aligned}
$$

where

$$
\begin{aligned}
& \chi_{n^{*} l m_{l}}(\zeta, \vec{r})=\frac{(2 \zeta)^{n^{*}+1 / 2}}{\sqrt{\Gamma\left(2 n^{*}+1\right)}} r^{n^{*}-1} e^{-\zeta r} S_{l m_{l}}(\theta, \varphi) \\
& \widetilde{\Pi}_{\mu u, \nu \sigma, n^{*} l m_{l}}^{\left(\delta^{*}\right) v}\left(\zeta, \zeta^{\prime} ; \vec{R}_{a b}\right)=\widetilde{\Omega}_{\mu \nu, u \nu}^{\left(\delta^{*}\right) v} \widetilde{\Lambda}_{\mu \nu \sigma, n^{*} l m_{l}}^{\left(\delta^{*}\right) v}\left(\zeta, \zeta^{\prime} ; \vec{R}_{a b}\right) .
\end{aligned}
$$

See (19) for definition of quantities $\widetilde{\Lambda}_{\mu \nu \sigma, n^{*} l m_{l}}^{\left(\delta^{*}\right) v}\left(\zeta, \zeta^{\prime} ; \vec{R}_{a b}\right)$ occurring in (29).

$$
\begin{aligned}
& \text { For } R_{a b}=0 \\
& \chi_{n^{*} l m_{l}}(\zeta, \vec{r}) \\
& \quad=\sum_{\mu=l+1}^{\infty} \sum_{u=l+1}^{\mu} \widetilde{\Pi}_{\mu u, l m_{l}, n^{*} l m_{l}}^{\left(\delta^{*}\right)}\left(\zeta, \zeta^{\prime}\right) \chi_{u-l \delta_{\delta^{*} p_{l}^{*}} l m_{l}}\left(\zeta^{\prime}, \vec{r}\right) .
\end{aligned}
$$

Here

$$
\begin{aligned}
& \widetilde{\Pi}_{\mu u, l m_{l}, n^{*} l m_{l}}^{\left(\delta^{*}\right) l}\left(\zeta, \zeta^{\prime}\right)=\widetilde{\Omega}_{\mu l, u l}^{\left(\delta^{*}\right) l} \widetilde{\Lambda}_{\mu l m_{l}, n^{*} l m_{l}}^{\left(\delta^{*}\right) l}\left(\zeta, \zeta^{\prime}\right) \\
& \widetilde{\Lambda}_{\mu l m_{l}, n^{*} l m_{l}}^{\left(\delta^{*}\right) l}\left(\zeta, \zeta^{\prime}\right) \\
& =\sum_{\mu^{\prime}=l+1}^{\mu} \tilde{a}_{\mu \mu^{\prime}}^{\left(\delta^{*}\right) l} \kappa_{\mu \mu^{\prime}}^{\left(\delta^{*}\right) l} S_{n^{*} l m_{l}, \mu^{\prime}+l \delta_{\delta^{*} p_{l}^{*}-\alpha^{*} l m_{l}}}\left(\zeta, \zeta^{\prime}\right),
\end{aligned}
$$

where

$$
\begin{aligned}
& \kappa_{\mu \mu^{\prime}}^{\left(\delta^{*}\right) l}=\sqrt{\Gamma\left(2\left(\mu^{\prime}+l-\alpha^{*}\right)+1\right)} \text { for } \delta^{*}=p_{l}^{*} \\
& \kappa_{\mu \mu^{\prime}}^{\left(\delta^{*}\right) l}=\sqrt{\frac{(2 \mu)^{\alpha^{*}} \Gamma\left(q_{\mu}^{*}+1\right) \Gamma\left(2\left(\mu^{\prime}-\alpha^{*}\right)+1\right)}{(\mu-(l+1)) !}} \quad \text { for } \delta^{*}=\alpha^{*} \\
& S_{n^{*} l_{l}, n^{\prime *} l^{\prime} m_{l}^{\prime}}^{*}\left(\zeta, \zeta^{\prime}\right) \\
& =\frac{\Gamma\left(n^{*}+n^{\prime *}+1\right)}{\sqrt{\Gamma\left(2 n^{*}+1\right) \Gamma\left(2 n^{\prime *}+1\right)}}(1+t)^{n^{*}+1 / 2} \\
& \cdot(1-t)^{n^{\prime *}+1 / 2} \cdot
\end{aligned}
$$

We note that the (27) and (30) are obtained by the use of complete orthogonal functions $\psi^{\left(\delta^{*}\right)}$-NSO.

\section{Quantum Nonrelativistic Field Theory in Nonstandard Convention}

Now we investigate the properties of nonrelativistic functions for $\alpha^{*}=1$ and $\zeta=Z / n$. In this case, these functions are determined by the following.

4.1. The Scalar Energies

$$
E^{\left(\delta^{*}\right)}=E=-\frac{Z^{2}}{2 n^{2}} .
$$

4.2. The Scalar Potentials

$$
V_{n l}^{\left(\delta^{*}\right)}(r)=V(r)=-\frac{Z}{r}
$$

4.3. The Scalar Forces

$$
F^{\left(\delta^{*}\right)}(r)=F(r)=-\frac{Z}{r^{2}}
$$

4.4. The Scalar Eigenfunctions

$$
\begin{aligned}
\psi_{n l m_{l}}^{\left(\delta^{*}\right)}(\zeta, \vec{r}) & =\psi_{n l m_{l}}\left(\frac{Z}{n}, \vec{r}\right) \\
& =\left(\frac{2 Z}{n}\right)^{3 / 2} e^{-(Z / n) r} L_{n l}^{p_{l}}\left(\frac{2 Z}{n} r\right) S_{l m_{l}}(\theta, \phi),
\end{aligned}
$$


where $L_{n l}^{p_{l}}((2 Z / n) r)$ are the associated Laguerre polynomials $\left(L^{p_{l}}\right.$-ALP) defined by

$$
\begin{aligned}
& \mathscr{L}_{n l}^{\left(\alpha^{*}\right)}(t)=L_{n l}^{p_{l}}\left(\frac{2 Z}{n} r\right) \\
& =\frac{(n+l) !}{(n-(l+1)) !}{ }_{1} F_{1}\left(-[n-(l+1)] ; p_{l}+1 ; t\right) \\
& \qquad \text { for } \alpha^{*}=1, \zeta=\frac{Z}{n} .
\end{aligned}
$$

Here $\varepsilon, V(r), F(r)$, and $\psi_{n l m_{l}}(Z / n, \vec{r})$ are the Schrödinger's eigenvalue, potential, force, and eigenfunction for the hydrogen-like atoms in nonstandard convention. As we see from (37), the $L^{p_{l}}$-ALP polynomials are the special cases of $\mathscr{L}^{\left(\delta^{*}\right)}$-NSSTP for $\alpha^{*}=1$ and $\zeta=Z / n$. The similar calculations can be also performed in the case of nonrelativistic standard convention. It should be noted that the eigenfunctions $\psi_{n l m_{l}}^{p_{l}}(Z / n, \vec{r})$ and Laguerre polynomials $L_{n l}^{p_{l}}((2 Z / n) r)$ obtained in nonrelativistic standard conventions are not complete basis sets. Therefore these functions cannot be used especially in the series expansion studies (see [17-20]).

\section{Quantum SF Relativistic NSST Field Theory}

In order to construct the complete orthogonal basis sets of SF relativistic $\Psi^{\left(\delta^{*}\right) s}$-RNSSTO and $X^{s}$-RSNSSTO orbitals, we use the properties of eigenfunctions of operators $\hat{j}^{2}, \hat{j}_{z}, \hat{l}^{2}$, and $\hat{s}^{2}$ which are determined by the following column-matrices $[21,22]$ :

$$
\begin{aligned}
&{ }^{1} Y_{j m_{j}}^{l s}(\theta, \phi)= {\left[\begin{array}{c}
a_{j m_{j}}^{l s}(0) Y_{l m_{l}(0)}(\theta, \phi) \\
a_{j m_{j}}^{l s}(1) Y_{l m_{l}(1)}(\theta, \phi) \\
\vdots \\
a_{j m_{j}}^{l s}(2 s-1) Y_{l m_{l}(2 s-1)}(\theta, \phi) \\
a_{j m_{j}}^{l s}(2 s) Y_{l m_{l}(2 s)}(\theta, \phi)
\end{array}\right] } \\
&{ }^{2} Y_{j m_{j}}^{l s}(\theta, \phi)=\left[\begin{array}{c}
a_{j m_{j}}^{l s}(2 s) Y_{l m_{l}(2 s)}(\theta, \phi) \\
a_{j m_{j}}^{l s}(2 s-1) Y_{l m_{l}(2 s-1)}(\theta, \phi) \\
\vdots \\
a_{j m_{j}}^{l s}(1) Y_{l m_{l}(1)}(\theta, \phi) \\
a_{j m_{j}}^{l s}(0) Y_{l m_{l}(0)}(\theta, \phi)
\end{array}\right] .
\end{aligned}
$$

Here $a_{j m_{j}}^{l s}(\lambda)=\left(l s m_{l}(\lambda) m_{s}(\lambda) / l s j m_{j}\right)$ are the Clebsch-Gordan coefficients [23], where

$$
\begin{aligned}
-l & \leq m_{l}(\lambda) \leq l, \\
-s & \leq m_{s}(\lambda) \leq s, \\
0 & \leq \lambda \leq 2 s, \\
m_{l}(\lambda) & =m_{j}-s+\lambda,
\end{aligned}
$$

$$
\begin{gathered}
|l-s| \leq j \leq l+s \\
-j \leq m_{j} \leq j .
\end{gathered}
$$

It is easy to show that the ${ }^{1} Y^{l s}$-RNSSTH and ${ }^{2} Y^{l s}$-MRNSSTH harmonics for $s=0$ are transformed into the scalar spherical harmonics:

$$
\begin{aligned}
& { }^{1} Y_{j m_{j}}^{l_{s}}(\theta, \phi)={ }^{2} Y_{j m_{j}}^{l_{s}}(\theta, \phi)=\left[\begin{array}{l}
S_{l m_{l}}(\theta, \phi) \\
S_{l m_{l}}(\theta, \phi)
\end{array}\right] \\
& \quad \text { for } s=0, j=l, m_{j}=m_{l},-l \leq m_{l} \leq l .
\end{aligned}
$$

It should be noted that (see $[21,22,24,25])$ the fermions (for $s=0,1 / 2,3 / 2, \ldots$ ) and bosons (for $s=1,2,3, \ldots$ ) are the special cases of SF particles when the SF properties disappear from the sight.

\subsection{Study of Properties of Quantum SF Relativistic NSST Func-} tions. The ${ }^{1} Y^{l s}$-RNSSTH and ${ }^{2} Y^{l s}$-MRNSSTH harmonics for fixed $s$ satisfy the following orthonormality relations $[21,22]$ :

$$
\begin{aligned}
& \int_{0}^{\pi} \int_{0}^{2 \pi}{ }^{1} Y_{j m_{j}}^{l s^{+}}(\theta, \phi)^{1} Y_{j^{\prime} m_{j}^{\prime}}^{l^{\prime} s}(\theta, \phi) \operatorname{Sin} \theta d \theta d \phi \\
& \quad=\delta_{l l^{\prime}} \delta_{j j^{\prime}} \delta_{m_{j} m_{j}^{\prime}} \\
& \int_{0}^{\pi} \int_{0}^{2 \pi}{ }^{2 \pi} Y_{j m_{j}}^{l s^{+}}(\theta, \phi)^{2} Y_{j^{\prime} m_{j}^{\prime}}^{l^{\prime} s}(\theta, \phi) \operatorname{Sin} \theta d \theta d \phi \\
& \quad=\delta_{l l^{\prime}} \delta_{j j^{\prime}} \delta_{m_{j} m_{j}^{\prime}} .
\end{aligned}
$$

Using (38)-(41), we obtain for Clebsch-Gordan coefficients the following properties:

$$
\sum_{\lambda=0}^{2 s}\left[a_{j m_{j}}^{l s}(\lambda) a_{j^{\prime} m_{j}^{\prime}}^{l s}(\lambda) \delta_{m_{l}(\lambda) m_{l}^{\prime}(\lambda)}\right] \delta_{l l^{\prime}}=\delta_{l l^{\prime}} \delta_{j j^{\prime}} \delta_{m_{j} m_{j}^{\prime}}
$$

where $-l \leq m_{l}(\lambda) \leq l$ and $0 \leq \lambda \leq 2 s$.

The SF relativistic NSST functions for arbitrary spin are defined by the following column-matrices:

$$
\begin{array}{r}
\Psi_{n l j m_{j}}^{\left(\delta^{*}\right) s}(\zeta, \vec{r}) \\
=\frac{1}{\sqrt{2}}\left[\begin{array}{c}
\widetilde{R}_{n l j}^{\left(\delta^{*}\right) s}(\zeta, r) a_{j m_{j}}^{l s}(0) Y_{l m_{l}(0)}(\theta, \phi) \\
\widetilde{R}_{n l j}^{\left(\delta^{*}\right) s}(\zeta, r) a_{j m_{j}}^{l s}(1) Y_{l m_{l}(1)}(\theta, \phi) \\
\vdots \\
\widetilde{R}_{n l j}^{\left(\delta^{*}\right) s}(\zeta, r) a_{j m_{j}}^{l s}(2 s) Y_{l m_{l}(2 s)}(\theta, \phi) \\
\widetilde{\Re}_{\tilde{n} l j}^{\left(\delta^{*}\right) s}(\zeta, r) a_{j m_{j}}^{l s}(2 s) Y_{l m_{l}(2 s)}(\theta, \phi) \\
\vdots \\
\widetilde{\mathfrak{R}}_{\tilde{n} l j}^{\left(\delta^{*}\right) s}(\zeta, r) a_{j m_{j}}^{l s}(1) Y_{l m_{l}(1)}(\theta, \phi) \\
\widetilde{\mathfrak{R}}_{\tilde{n} l j}^{\left(\delta^{*}\right) s}(\zeta, r) a_{j m_{j}}^{l s}(0) Y_{l m_{l}(0)}(\theta, \phi)
\end{array}\right] \\
\text { for } s=\frac{1}{2}, 1, \frac{3}{2}, 2, \ldots
\end{array}
$$




$$
\begin{aligned}
& \Psi_{n l j m_{j}}^{\left(\delta^{*}\right) s}(\zeta, \vec{r}) \\
& =\frac{1}{\sqrt{2}}\left[\begin{array}{l}
\widetilde{R}_{n l j}^{\left(\delta^{*}\right) s}(\zeta, r) a_{j m_{j}}^{l s}(0) Y_{l m_{l}(0)}(\theta, \phi) \\
\widetilde{\Re}_{\widetilde{n} l j}^{\left(\delta^{*}\right) s}(\zeta, r) a_{j m_{j}}^{l s}(0) Y_{l m_{l}(0)}(\theta, \phi)
\end{array}\right] \\
& \text { for } s=0, j=l, m_{j}=m_{l}(0),-l \leq m_{l}(0) \leq l \\
& \bar{\Psi}_{n l j m_{j}}^{\left(\delta^{*}\right) s}(\zeta, \vec{r})=\beta_{n l}^{\left(\delta^{*}\right)}(t) \Psi_{n l j m_{j}}^{\left(\delta^{*}\right) s}(\zeta, \vec{r}) \\
& \text { for } s=\frac{1}{2}, 1, \frac{3}{2}, 2, \ldots \\
& \bar{\Psi}_{n l j m_{j}}^{\left(\delta^{*}\right) s}(\zeta, \vec{r})=\beta_{n l}^{\left(\delta^{*}\right)}(t) \Psi_{n l j m_{j}}^{\left(\delta^{*}\right) s}(\zeta, \vec{r}) \\
& \text { for } s=0, j=l, m_{j}=m_{l}(0),-l \leq m_{l}(0) \leq l \\
& X_{n l j m_{j}}^{s}(\zeta, \vec{r}) \\
& =\frac{1}{\sqrt{2}}\left[\begin{array}{c}
\widetilde{R}_{n l j}^{\left(\delta^{*}\right) s}(\zeta, r) a_{j m_{j}}^{l s}(0) Y_{l m_{l}(0)}(\theta, \phi) \\
\widetilde{R}_{n l j}^{\left(\delta^{*}\right) s}(\zeta, r) a_{j m_{j}}^{l s}(1) Y_{l m_{l}(1)}(\theta, \phi) \\
\vdots \\
\widetilde{R}_{n l j}^{\left(\delta^{*}\right) s}(\zeta, r) a_{j m_{j}}^{l s}(2 s) Y_{l m_{l}(2 s)}(\theta, \phi) \\
\widetilde{\mathfrak{R}}_{\tilde{n} l j}^{\left(\delta^{*}\right) s}(\zeta, r) a_{j m_{j}}^{l s}(2 s) Y_{l m_{l}(2 s)}(\theta, \phi) \\
\vdots \\
\widetilde{\mathfrak{R}}_{\tilde{n} l j}^{\left(\delta^{*}\right) s}(\zeta, r) a_{j m_{j}}^{l s}(1) Y_{l m_{l}(1)}(\theta, \phi) \\
\widetilde{\mathfrak{R}}_{\widetilde{n} l j}^{\left(\delta^{*}\right) s}(\zeta, r) a_{j m_{j}}^{l s}(0) Y_{l m_{l}(0)}(\theta, \phi)
\end{array}\right] \\
& \text { for } s=\frac{1}{2}, 1, \frac{3}{2}, 2, \ldots \\
& X_{n l j m_{j}}^{s}(\zeta, \vec{r}) \\
& =\frac{1}{\sqrt{2}}\left[\begin{array}{c}
\widetilde{R}_{n l j}^{\left(\delta^{*}\right) s}(\zeta, r) a_{j m_{j}}^{l s}(0) Y_{l m_{l}(0)}(\theta, \phi) \\
\widetilde{\Re}_{\tilde{n} l j}^{\left(\delta^{*}\right) s}(\zeta, r) a_{j m_{j}}^{l s}(0) Y_{l m_{l}(0)}(\theta, \phi)
\end{array}\right] \\
& \text { for } s=0, j=l, m_{j}=m_{l}(0),-l \leq m_{l}(0) \leq l \text {, }
\end{aligned}
$$

where $n \geq 1, s \leq j \leq s+n-1, j-s \leq l \leq \min (j+s, n-$ $1), \widetilde{n}=n+2|j-l|$. See (53) and $[21,22,24-32]$ for the exact definition of functions occurring in ((43a) and (43b))-((45a) and (45b)).

The relativistic orbitals of arbitrary $\operatorname{spin}(s=0,1 / 2,1$, $3 / 2,2, \ldots)$ and nonrelativistic functions are determined by the following relations:

$$
\Psi_{n l j m_{j}}^{\left(\delta^{*}\right) s}(\zeta, \vec{r})=\Psi_{n l j m_{j}}^{\left(\delta^{*}\right)}(\zeta, \vec{r})\left[\begin{array}{c}
1 \\
1 \\
\vdots \\
1 \\
1 \\
\vdots \\
1 \\
1
\end{array}\right]
$$

$$
\begin{aligned}
& \Psi_{n l j m_{j}}^{\left(\delta^{*}\right) s}(\zeta, \vec{r})=\psi_{n l m_{l}(0)}^{\left(\delta^{*}\right)}(\zeta, \vec{r}) \\
& \text { for } j=l, m_{j}=m_{l}(0),-l \leq m_{l}(0) \leq l \\
& \bar{\Psi}_{n l j m_{j}}^{\left(\delta^{*}\right) s}(\zeta, \vec{r})=\beta_{n l}^{\left(\delta^{*}\right)}(t) \Psi_{n l j m_{j}}^{\left(\delta^{*}\right) s}(\zeta, \vec{r}) \\
& \Psi_{n l j m_{j}}^{\left(\delta^{*}\right)}(\zeta, \vec{r})=\psi_{n l m_{l}(0)}^{\left(\delta^{*}\right)}(\zeta, \vec{r}) \\
& \text { for } j=l, m_{j}=m_{l}(0),-l \leq m_{l}(0) \leq l \\
& X_{n l j m_{j}}^{s}(\zeta, \vec{r})=X_{n l j m_{j}}(\zeta, \vec{r})\left[\begin{array}{c}
1 \\
1 \\
\vdots \\
1 \\
1 \\
\vdots \\
1 \\
1
\end{array}\right] \\
& X_{n l j m_{j}}(\zeta, \vec{r})=\chi_{n l m_{l}(0)}(\zeta, \vec{r}) \\
& \text { for } j=l, m_{j}=m_{l}(0),-l \leq m_{l}(0) \leq l \text {. }
\end{aligned}
$$

Using ((46a) and (46b)) $-((48 a)$ and $(48 b))$ it is easy to show that the SF relativistic NSST functions for spin $s=0$ and $j=l$ simple are reduced to the corresponding nonrelativistic scalar basis sets:

$$
\begin{aligned}
& \Psi_{1000}^{\left(\delta^{*}\right) 0}(\zeta, \vec{r})=\left[\begin{array}{l}
\Psi_{1000}^{\left(\delta^{*}\right)}(\zeta, \vec{r}) \\
\Psi_{1000}^{\left(\delta^{*}\right)}(\zeta, \vec{r})
\end{array}\right] \\
& \Psi_{1000}^{\left(\delta^{*}\right)}(\zeta, \vec{r})=\psi_{100}^{\left(\delta^{*}\right)}(\zeta, \vec{r}) \\
& \Psi_{1000}^{\left(\delta^{*}\right) 0}(\zeta, \vec{r})=\left[\begin{array}{l}
\Psi_{1000}^{\left(\delta^{*}\right)}(\zeta, \vec{r}) \\
\Psi_{1000}^{\left(\delta^{*}\right)}(\zeta, \vec{r})
\end{array}\right] \\
& \Psi_{1000}^{\left(\delta^{*}\right)}(\zeta, \vec{r})=\bar{\psi}_{100}^{\left(\delta^{*}\right)}(\zeta, \vec{r}) \\
& X_{1000}^{0}(\zeta, \vec{r})=\left[\begin{array}{l}
X_{1000}(\zeta, \vec{r}) \\
X_{1000}(\zeta, \vec{r})
\end{array}\right] \\
& X_{1000}(\zeta, \vec{r})=\chi_{100}(\zeta, \vec{r}) .
\end{aligned}
$$

The orthogonality relations for SF relativistic NSST functions are determined by

$$
\begin{aligned}
& \int \Psi_{n l j m_{j}}^{\left(\delta^{*}\right) s^{\dagger}}(\zeta, \vec{r}) \bar{\Psi}_{n^{\prime} l^{\prime} j^{\prime} m_{j}^{\prime}}^{\left(\delta^{*}\right) s}(\zeta, \vec{r}) d^{3} \vec{r}=\delta_{n n^{\prime}} \delta_{l l^{\prime}} \delta_{j j^{\prime}} \delta_{m_{j} m_{j}^{\prime}} \\
& \int X_{n l j m_{j}}^{s^{\dagger}}(\zeta, \vec{r}) X_{n^{\prime} l^{\prime} j^{\prime} m_{j}^{\prime}}^{s}(\zeta, \vec{r}) d^{3} \vec{r}=\delta_{n n^{\prime}} \delta_{l l^{\prime}} \delta_{j j^{\prime}} \delta_{m_{j} m_{j}^{\prime}},
\end{aligned}
$$


where $n \geq 1, s \leq j \leq s+n-1,-j \leq m_{j} \leq j, j-s \leq l \leq$ $\min (j+s, n-1)$ and

$$
\begin{gathered}
\widetilde{R}_{n l j}^{\left(\delta^{*}\right) s}(\zeta, r)=R_{n l}^{\left(\delta^{*}\right)}(\zeta, r), \\
\widetilde{\mathfrak{R}}_{\tilde{n} l j}^{\left(\delta^{*}\right) s}(\zeta, r)=R_{\tilde{n} l}^{\left(\delta^{*}\right)}(\zeta, r), \\
\widetilde{\bar{R}}_{n l j}^{\left(\delta^{*}\right) s}(\zeta, r)=\bar{R}_{n l}^{\left(\delta^{*}\right)}(\zeta, r), \\
\widetilde{\overline{\mathfrak{R}}}_{\widetilde{n} l j}^{\left(\delta^{*}\right) s}(\zeta, r)=\bar{R}_{\tilde{n} l}^{\left(\delta^{*}\right)}(\zeta, r), \\
\widetilde{R}_{n l j}^{s}(\zeta, r)=R_{n l}(\zeta, r), \\
\widetilde{\mathfrak{R}}_{\tilde{n} l j}^{s}(\zeta, r)=R_{\widetilde{n} l}(\zeta, r),
\end{gathered}
$$

where $R_{n l}^{\left(\delta^{*}\right)}(\zeta, r), \quad R_{\tilde{n} l}^{\left(\delta^{*}\right)}(\zeta, r), \quad \bar{R}_{n l}^{\left(\delta^{*}\right)}(\zeta, r), \quad \bar{R}_{\tilde{n} l}^{\left(\delta^{*}\right)}(\zeta, r) \quad$ and $R_{n l}(\zeta, r)=R_{n}(\zeta, r), R_{\widetilde{n l}}(\zeta, r)=R_{\widetilde{n}}(\zeta, r)$ functions are the radial parts of nonrelativistic scalar orbitals $\psi_{n l m_{l}}^{\left(\delta^{*}\right)}(\zeta, \vec{r})$, $\bar{\psi}_{n l m_{l}}^{\left(\delta^{*}\right)}(\zeta, \vec{r})$, and $\chi_{n l m_{l}}(\zeta, \vec{r})$, respectively.

\subsection{The SF Relativistic NSST and Nonrelativistic Energies}

$$
\begin{aligned}
& E_{n l j m_{j}}^{S}(\zeta)=E_{n l j m_{j}}(\zeta) I^{2(2 s+1)} \\
& E_{n l j m_{j}}(\zeta)=E_{n l}(\zeta)=-\frac{\zeta^{2}}{2} .
\end{aligned}
$$

\subsection{The SF Relativistic NSTT and Nonrelativistic Potentials} and Their Derivatives

$$
\begin{aligned}
V_{n l j m_{j}}^{\left(\delta^{*}\right) s}(\zeta, r) & =V_{n l j m_{j}}^{\left(\delta^{*}\right)}(\zeta, r) I^{2(2 s+1)} \\
\frac{d^{k}}{d r^{k}} V_{n l j m_{j}}^{\left(\delta^{*}\right) s}(\zeta, r) & =\frac{d^{k}}{d r^{k}} V_{n l j m_{j}}^{\left(\delta^{*}\right)}(\zeta, r) I^{2(2 s+1)} \\
\frac{d^{k}}{d r^{k}} V_{n l j m_{j}}^{\left(\delta^{*}\right) s}(\zeta, r) & =\frac{d^{k}}{d r^{k}} V_{n l}^{\left(\delta^{*}\right)}(\zeta, r),
\end{aligned}
$$

where $1 \leq k<\infty$.

The (55a), (55b), and (55c) describe the features of quantum SF relativistic NSST nonperturbative theory. In the case of non-self-frictional (NSF) relativistic field, the nonperturbative approach is reduced to the perturbation series which are divergent in Quantum Electrodynamics (see [33-35]).

In the literature (see [33-37] and references therein), the NSF relativistic nonperturbative theory has been considered. We notice that the radiation and self-force problems arise in the NSF nonperturbative theory presented in [33-37].

As an application of quantum SF relativistic NSST nonperturbative theory, the comparative values of scalar and SF relativistic NSST potentials $V^{\left(\delta^{*}\right)}(\zeta, r)$ and forces $F^{\left(\delta^{*}\right)}(\zeta, \vec{r})=$ $-(d / d r) V^{\left(\delta^{*}\right)}(\zeta, r)$ for $\delta^{*}=\alpha^{*}$ and $10^{-18} \leq r \leq 10^{4}$ are shown in Figures 1 and 2.

\section{Conclusion}

The construction of quantum self-frictional relativistic nucleoseed spinor-type tensor field theory of nature is based on the generalization of AMN approach to the CU systems introduced by the author in the previous papers. It has been shown that the gravitational, nuclear $\left(N_{1}\right.$ and $\left.N_{2}\right)$, electromagnetic, nuclear weak, and Newtonian fields are the special cases of quantum SF relativistic NSST field presented in this work. We note that the fermions and bosons are obtained from the SF particles of arbitrary $\operatorname{spin}(s=0,1 / 2,1,3 / 2,2, \ldots)$ when their SF properties disappear.

The one- and two-center one-range addition theorems for nonrelativistic noninteger $n \chi$-NSTO orbitals have been suggested. The SF relativistic NSST field through the nonrelativistic field, and vice versa, has been presented. The quantum self-frictional relativistic nonperturbative theory has been also suggested.

The anomaly in $[3,4]$ could lead to a fundamental revision of the Quantum Electrodynamics theory known as the besttested and best-understood theory in all of science until now. We believe that the presented quantum SF relativistic NSST field approach will be of interest in the quantum mechanics of cosmic sciences and combined open shell Hartree-Fock theory suggested by the author (see [38] and references therein to our papers).

The application of presented theory is in progress in our group for the study of SF-NSST atomic-molecular-nuclear and cosmic-universe systems.

\section{Competing Interests}

The author declares that he has no competing interests.

\section{References}

[1] H. F. M. Goenner, "On the history of unified field theories," Living Reviews in Relativity, vol. 7, no. 2, pp. 5-129, 2004.

[2] H. F. M. Goenner, "On the history of unified field theories, part II. (ca. 1930-ca1965)," Living Reviews in Relativity, vol. 17, no. 5, pp. 5-241, 2014.

[3] J. C. Bernauer and R. Pohl, “The proton radius problem," Scientific American, vol. 310, no. 2, pp. 32-39, 2014.

[4] B. P. Abbott, R. Abbott, T. D. Abbott et al., "Observation of gravitational waves from a binary black hole merger," Physical Review Letters, vol. 116, no. 6, Article ID 061102, 16 pages, 2016.

[5] H. A. Lorentz, The Theory of Electrons, Dover, New York, NY, USA, 1953.

[6] W. Heitler, The Quantum Theory of Radiation, Oxford University Press, 1950.

[7] L. D. Landau and E. M. Lifshitz, The Classical Theory of Fields, Pergamon, New York, NY, USA, 1987.

[8] I. I. Guseinov, "New complete orthonormal sets of exponentialtype orbitals in standard convention and their origin," Bulletin of the Chemical Society of Japan, vol. 85, no. 12, pp. 1306-1309, 2012.

[9] I. I. Guseinov, "One-range addition theorems for noninteger $\mathrm{n}$ slater functions using complete orthonormal sets of exponential type orbitals in standard convention," Few-Body Systems, vol. 54, no. 11, pp. 1773-1780, 2013. 
[10] I. I. Guseinov, "Unified treatment of one-range addition theorems for complete orthonormal sets of generalized exponentialtype orbitals and noninteger N slater functions," Bulletin of the Chemical Society of Japan, vol. 87, no. 10, pp. 1101-1103, 2014.

[11] I. I. Guseinov, "Combined open shell Hartree-Fock theory of atomic-molecular and nuclear systems," Journal of Mathematical Chemistry, vol. 42, no. 2, pp. 177-189, 2007.

[12] B. A. Mamedov, "Israfil I. Guseinov: a pioneer of the quantum theory of atomic, molecular, and nuclear systems," International Journal of Quantum Chemistry, vol. 114, no. 5, pp. 361-366, 2014.

[13] I. I. Guseinov, "Analytical evaluation of two-centre Coulomb, hybrid and one-electron integrals for Slater-type orbitals," Journal of Physics B: Atomic and Molecular Physics, vol. 3, no. 11, pp. 1399-1412, 1970.

[14] E. U. Condon and G. H. Shortley, The Theory of Atomic Spectra, Cambridge University Press, Cambridge, UK, 1970.

[15] W. Magnus, F. Oberhettinger, and R. P. Soni, Formulas and Theorems for the Special Functions of Mathematical Physics, Springer, New York, NY, USA, 1966.

[16] I. I. Guseinov and B. A. Mamedov, "On the accurate evaluation of overlap integrals over slater type orbitals using analytical and recurrence relations," Zeitschrift fur Naturforschung, vol. 62, no. 9, pp. 467-470, 2007.

[17] E. A. Hylleraas, "A new calculation of the energy of helium in the ground state as well as the lowest term of ortho-helium," Zeitschrift für Physik, vol. 54, pp. 347-355, 1929.

[18] E. A. Hylleraas, "On the ground state term of the two-electron problem of $\mathrm{H}^{-}, \mathrm{He}, \mathrm{Li}^{+}, \mathrm{Be}^{++}$, etc," Zeitschrift für Physik, vol. 65, pp. 209-221, 1930.

[19] H. Shull and P.-O. Löwdin, "Role of the continuum in superposition of configurations," The Journal of Chemical Physics, vol. 23, no. 7, p. 1362, 1955.

[20] P.-O. Löwdin and H. Shull, "Natural orbitals in the quantum theory of two-electron systems," Physical Review, vol. 101, no. 6, pp. 1730-1739, 1956.

[21] I. I. Guseinov, "Unified treatment of complete orthonormal sets for wave functions, and Slater orbitals of particles with arbitrary spin in coordinate, momentum and four-dimensional spaces," Physics Letters A, vol. 372, no. 1, pp. 44-48, 2007.

[22] I. I. Guseinov, "Theory of complete orthonormal sets of relativistic tensor wave functions and Slater tensor orbitals of particles with arbitrary spin in position, momentum and four-dimensional spaces," Physics Letters A, vol. 373, no. 25, pp. 2178-2181, 2009.

[23] I. A. R. Edmons, Angular Momentum in Quantum Mechanics, Princeton University Press, Princeton, NJ, USA, 1957.

[24] I. I. Guseinov, "Dirac equation for particles with arbitrary halfintegral spin," Philosophical Magazine, vol. 91, no. 31, pp. 40634072, 2011.

[25] I. I. Guseinov, "Unified treatment of basis sets of relativistic exponential-type spinor orbitals for particles with arbitrary halfintegral spin in position, momentum and four-dimensional spaces," Physica Scripta, vol. 86, no. 5, 2012.

[26] I. I. Guseinov, "New complete orthonormal sets of exponentialtype orbitals and their application to translation of slater orbitals," International Journal of Quantum Chemistry, vol. 90, no. 1, pp. 114-118, 2002.

[27] I. I. Guseinov, "Addition and expansion theorems for complete orthonormal sets of exponential-type orbitals in coordinate and momentum representations," Journal of Molecular Modeling, vol. 9, no. 3, pp. 135-141, 2003.
[28] I. I. Guseinov, "New complete orthonormal sets of hyperspherical harmonics and their one-range addition and expansion theorems," Journal of Molecular Modeling, vol. 12, no. 6, pp. 757761, 2006.

[29] I. I. Guseinov, "New developments in quantum mechanics and applications," AIP Conference Proceedings, vol. 889, pp. 65-68, 2007.

[30] I. I. Guseinov, "Unified treatment of complete orthonormal sets of functions in coordinate, momentum and four-dimensional spaces and their expansion and one-range addition theorems," Journal of Mathematical Chemistry, vol. 42, no. 4, pp. 991-1001, 2007.

[31] I. I. Guseinov, "Unified treatment of complete orthonormal sets of nonrelativistic, quasirelativistic and relativistic sets of spinor wave functions, and Slater spinor orbitals in coordinate, momentum and four-dimensional spaces," Journal of Mathematical Chemistry, vol. 44, no. 3, pp. 839-848, 2008.

[32] I. I. Guseinov, "Combined theory of two- and four-component complete orthonormal sets of spinor wave functions and Slater type spinor orbitals in position, momentum and four-dimensional spaces," Journal of Mathematical Chemistry, vol. 47, no. 1, pp. 391-402, 2010.

[33] F. J. Dyson, "Divergence of perturbation theory in quantum electrodynamics," Physical Review, vol. 85, no. 4, pp. 631-633, 1952.

[34] I. W. Herbst and B. Simon, "Some remarkable examples in eigenvalue perturbation theory," Physics Letters B, vol. 78, no. 2, pp. 304-306, 1978.

[35] C. M. Bender and K. A. Milton, "A nonunitary version of massless quantum electrodynamics possessing a critical point," Journal of Physics A, vol. 32, no. 7, p. L87, 1999.

[36] D. J. Gross, "Non-perturbative string theory," in Random Surfaces and Quantum Gravity, vol. 262 of NATO ASI Series, pp. 255-267, Springer, Berlin, Germany, 1991.

[37] Y. Frishman and J. Sonnenschein, Non-Perturbative Field Theory, Cambridge University Press, 2010.

[38] I. I. Guseinov, "Combined open shell Hartree-Fock theory of atomic and molecular systems constructed from noncharged scalar particles," Physics Essays, vol. 27, no. 3, pp. 351-355, 2014. 

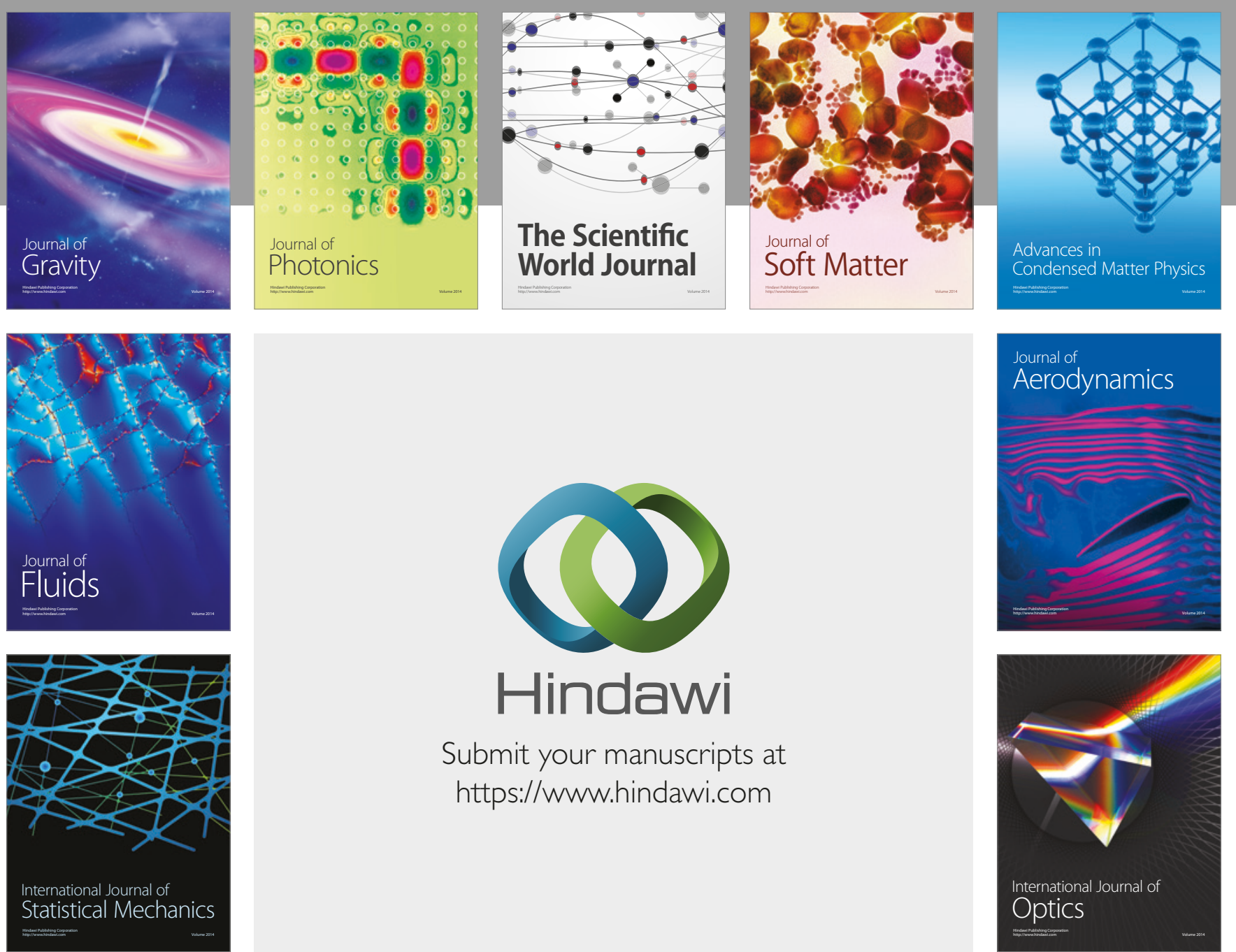

Submit your manuscripts at

https://www.hindawi.com
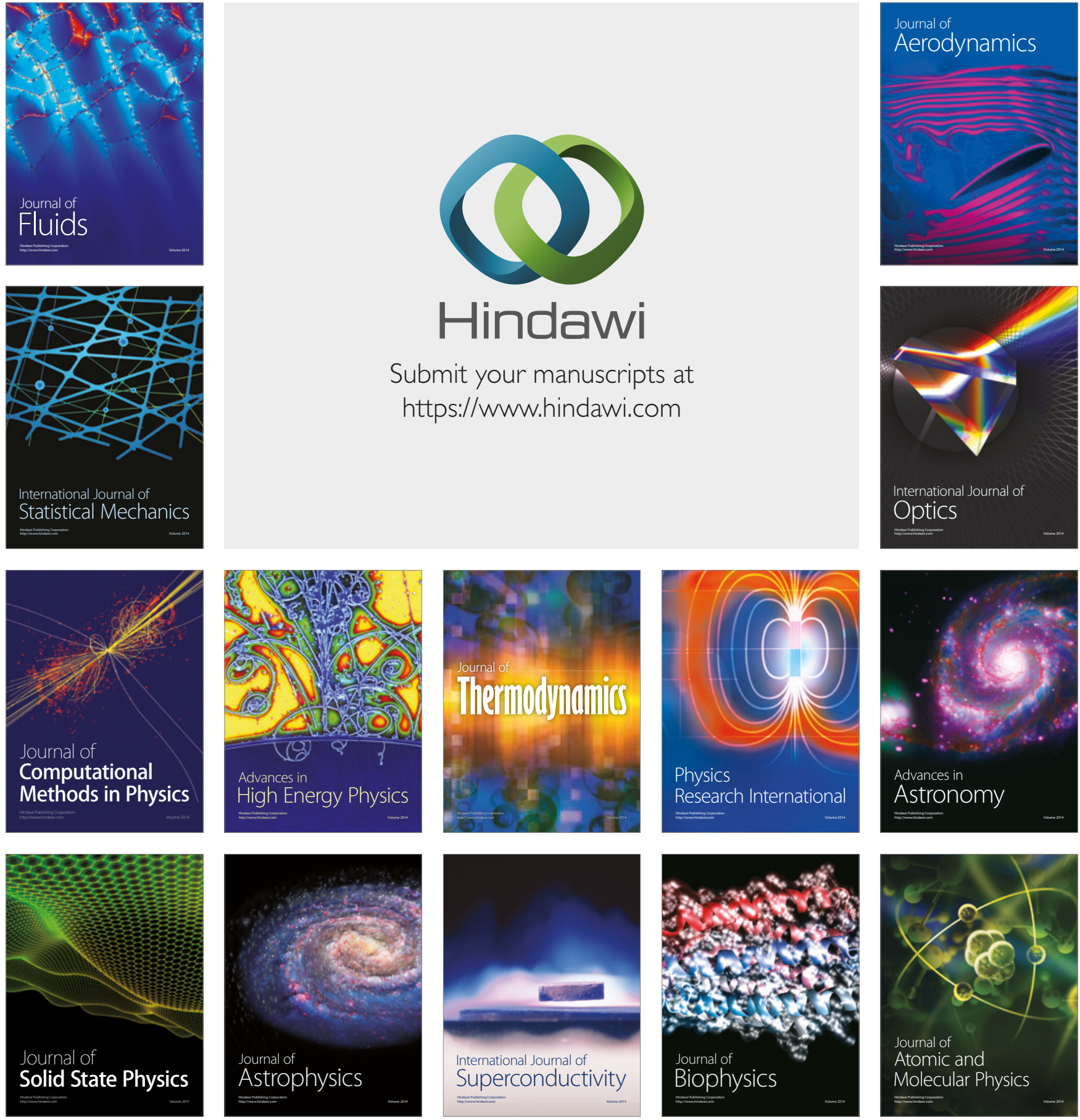